\title{
Clases y reproducción social: el espacio social cordobés en la primera década del siglo XXI
}

\author{
Alicia B. GutiérReZ \\ CONICET - Universidad Nacional de Córdoba \\ gutierre@ffyh.unc.edu.ar
}

\author{
Héctor MansiLla \\ Universidad Nacional de Córdoba \\ hectorosvaldomansillla@gmail.com
}

Recibido: 18-02-2014

Aceptado: 26-02-2015

\section{Resumen}

En este artículo proponemos una construcción del espacio social del Gran Córdoba (Argentina) en la última década, como primer momento (objetivista) de una investigación sobre las estrategias de reproducción social de las distintas clases y fracciones de clase que lo componen.

Ello supone hacer una elección teórica respecto a la manera de concebir las clases sociales y su conjunto y proponer una articulación teórico-metodológica para su construcción empírica. Asimismo, conlleva una serie de decisiones técnicas destinadas a adecuar esa articulación con la utilización de las fuentes de datos del sistema estadístico nacional.

Partiendo de la noción bourdieusiana de espacio social, proponemos una construcción relacional de las clases y fracciones a partir del conjunto de recursos - fundamentalmente económicos y culturales - que disponen las familias para su reproducción. Para ello, apelamos a la utilización de métodos de estadística descriptiva multidimensional conforme a la escuela francesa de análisis de datos. Aplicamos de manera conjunta métodos factoriales y de clasificación, tomando como base la información captada por la "Encuesta Permanente de Hogares" en los terceros trimestres de los años 2003 y 2011.

La construcción del espacio social, que identifica cuatro grandes clases con sus respectivas fracciones y muestra una estructura homóloga en ambos períodos, nos permitirá explicitar el debate teórico en torno a las clases y su dinámica, mostrar el valor heurístico de las decisiones tomadas previamente, $\mathrm{y}$ las condiciones de posibilidad para poner en marcha la etapa cualitativa de la investigación.

Palabras clave: espacio social, clases, fracciones de clase, estrategias, reproducción social. 


\title{
Class and Social Reproduction: the Social Space in Córdoba at the First Decade of the Twenty First Century
}

\begin{abstract}
In this paper we propose a construction of the social space of Gran Córdoba (Argentina) in the last decade, as a first moment (objectivist) of a research about social reproduction's strategies of the different classes and class fractions that compose it.

This implies a theoretical choice about the way to conceive the social classes and the dynamics of their reproduction. It also means to propose a theoretical and methodological articulation for its empirical construction. It also entails a series of technical decisions to adapt this articulation with the use of the data sources from the national statistical system.

Based on the notion of social space (Bourdieu), we propose a relational construction of classes and fractions from the set of family resources available for their social reproduction, mainly economical y cultural. For this construction we apply jointly factorial methods and classificatory methods, based on the information captured by the "Encuesta Permanente de Hogares" in the third quarters of 2003 and 2011.

The construction of social space, which identifies four main classes with their respective fractions and shows a homologous structure in both periods, will allow us to make explicit the theoretical debate about the classes and their dynamics, to show the heuristic value of the decisions previously made, and it is the conditions of possibility for implementing the qualitative stage of the research.
\end{abstract}

Keywords: Social space; Class; Class fractions; Strategies; Social reproduction.

\section{Referencia normalizada}

Gutiérrez, Alicia B. y Héctor Mansilla (2015): “Clases y reproducción social:el espacio social cordobés en la primera década del siglo XXI”, Política y Sociedad, 52 (2), pp. 409-441.

Sumario: 1. Introducción: espacio social, reproducción y estrategias. 2. La construcción del espacio social cordobés de la última década. 3. Homología estructural y trayectorias. 4. Reflexiones finales: puntos de partida y apuestas. 5. Bibliografía. 


\section{Introducción: espacio social, reproducción y estrategias}

En este artículo construimos el espacio social del Gran Córdoba ${ }^{1}$ como primer momento objetivista — en el sentido de Bourdieu et al. (1975) — de una investigación sobre estrategias de reproducción de familias de las diferentes clases sociales que lo componen. Pretendemos dar cuenta de la articulación entre esas prácticas y la estructuracióntransformación del espacio social cordobés en el decenio 2003-2013, período signado por la relativa estabilidad conseguida luego de la crisis $2001-2002^{2}$.

Partimos del supuesto de que la vida social (con sus relaciones de desigualdad y dominación) se produce y se reproduce a través de las prácticas, como resultado de la relación dialéctica entre estructuras y agentes, en el sentido en que lo proponen Bourdieu $(1988,1990)$ y Giddens $(1987,1995)$. En ese marco, un concepto central es el de estrategias de reproducción social, entendidas como "conjunto de prácticas fenomenalmente muy diferentes, por medio de las cuales los individuos y las familias tienden, de manera consciente o inconsciente, a conservar o a aumentar su patrimonio, y correlativamente a mantener o mejorar su posición en la estructura de las relaciones de clase" (Bourdieu, 1988: 122).

Dichas estrategias dependen de un conjunto de factores: 1) del volumen y la estructura del capital que hay que reproducir (es decir, los diferentes tipos de recursos con los que cuentan las familias: capital económico, capital cultural, capital social, capital simbólico) y de su trayectoria histórica; 2) del estado del sistema de los instrumentos de reproducción o de las condiciones objetivas donde se insertan estas prácticas, tales como el mercado laboral, el mercado escolar, el mercado de la vivienda, el mercado del consumo cultural, etc.; 3) del estado de la relación de fuerzas entre las clases, es decir de los límites y posibilidades ligadas a las familias de las diferentes clases $-\mathrm{y}$ fracciones de clase- por su reproducción conjunta en el mismo espacio social y 4) de los habitus

${ }^{1}$ El Instituto Nacional de Estadísticas y Censos considera "Gran Córdoba" a la conurbación de la ciudad de Córdoba (Argentina), que comprende no solamente la ciudad de Córdoba capital, sino también un conjunto de localidades del Departamento Colón. Según el Censo 2010, cuenta con 1.412.182 habitantes, constituyendo la segunda aglomeración urbana del país.

${ }^{2}$ Esta crisis mostró con crudeza la realidad social argentina que había empezado a construirse a mediados de la década de 1970 y que se consolidó en la de 1990, caracterizada por la implementación de políticas de corte neoliberal y la retracción del Estado. A partir de 2003, la orientación política y económica del gobierno kirschnerista modificó el rol del Estado, aumentando su presencia a través de políticas de inclusión social (previsionales, educativas ) además de propiciar medidas que impulsaron la reindustrialización del país, logrando una fuerte disminución de los índices de desempleo y de pobreza. 
incorporados por los agentes sociales, es decir, de su sistema de disposiciones a actuar, a percibir y a evaluar esas posibilidades y límites. (Gutiérrez, 2007)³.

La unidad de análisis principal es la familia - considerada como unidad doméstica- y el punto de partida del estudio es su posición en la estructura de clases, tomando en cuenta la totalidad y el valor relativo de sus recursos. La construcción del espacio social cordobés se impone pues como primer paso de la investigación y en él se centra nuestro artículo.

Primero, en el marco general del debate acerca de las clases sociales y su dinámica, explicitaremos la perspectiva teórica que sustenta nuestra propuesta y el modo como ésta se articula con la metodología seleccionada, junto a las decisiones técnicas tomadas para adecuar estas dimensiones a las fuentes de información utilizadas. Luego describiremos el espacio social cordobés para $2011^{4}$, caracterizando sus regiones y las clases y fracciones que lo conforman, ofreciendo diagramas y tablas que permiten visualizarlo. Después, reconstruiremos el espacio social para 2003, mostrando las homologías estructurales y formulando algunas hipótesis sobre su trayectoria; finalmente, este trabajo empírico nos permitirá recuperar nuestra posición en el debate sobre las clases y su dinámica y mostrar el valor heurístico de las decisiones teórico-metodológicas y técnicas tomadas.

\section{La construcción del espacio social cordobés de la última década}

\subsection{La perspectiva teórica seleccionada: herramientas conceptuales}

Los enfoques más establecidos en el terreno de estudio de las clases sociales han tendido a centrarse en aspectos económicos para su definición, atendiendo a dimensiones como la ocupación, las relaciones de producción, los niveles de ingresos. Con frecuencia se ha prescindido de otros elementos para comprender las dinámicas de las clases sociales, como pueden ser los elementos simbólicos. Los trabajos de los dos autores de referencia en los estudios de movilidad y estratificación social, como Goldthorpe y E. $\mathrm{O}$. Wright, toman como relevante para su estudio la dimensión económica, manteniendo la diferenciación weberiana entre clase y grupo de status (Crompton, 1997).

Estos enfoques, que desarrollaron sus propuestas en oposición al tratamiento funcionalista de la estratificación social (la que desconoce completamente las relaciones de poder y de conflicto en la sociedad), están sustentados respectivamente en las teorías

${ }^{3}$ Un conjunto de investigaciones llevadas adelante desde esta perspectiva revelan la fertilidad del enfoque teórico, a la vez que la pertinencia de tomar como unidades de análisis tanto a las familias como a las redes sociales (Gutiérrez, 2004, 2005 y 2008; Jiménez Zunino, 2011a; Capdevielle, 2012; Freyre, 2013).

${ }^{4} 2011$ corresponde a la disponibilidad de fuentes al momento de comenzar nuestro estudio. 
de Weber y Marx. Así, tomando los conceptos weberianos de situación de mercado y situación de trabajo, Goldthorpe (1994) clasifica las diferentes categorías ocupacionales, de acuerdo con sus oportunidades de vida propiciando distintas situaciones de clase (Giddens, 1983; Crompton, 1997.; Jorrat, 2005). Erik Olin Wright por su parte, rechazando la identificación de la movilidad social con la movilidad ocupacional, propone un esquema marxista de clases en el que no sólo estarían capitalistas y proletarios, sino que también ubica lo que denomina "posiciones contradictorias de clase" (Wright, 1994), en las que sitúa a las clases medias.

Podemos decir que ambas corrientes ("marxistas" y "weberianas") presentan, al menos, dos limitaciones desde el punto de vista teórico: a) tienden a tratar a las clases como sustancias dadas o entidades "preconstruidas en la estructura social, sea sobre la base de criterios puramente económicos, o sobre fundamentos de autoridad, credenciales y relaciones de mercado" (Wacquant, 1991.: 50) y b) no pueden relacionar adecuadamente las clases y la cultura ${ }^{6}$, los aspectos económicos y no económicos que definen y distinguen diferentes posiciones sociales a las que se encuentran asociadas diferentes "estilos de vida".

En los estudios latinoamericanos, las perspectivas analíticas más utilizadas en la actualidad se corresponden con los modelos más asentados. Entre ellos, destacamos los análisis sobre la movilidad ocupacional en el Cono Sur (Espinoza, 2006), los estudios realizados en Argentina por Jorrat (2005) — que suponen una síntesis entre diversas aportaciones teóricas (de Erikson, de Goldthorpe y de Portocarero) para brindar una categorización de los grupos ocupacionales y las dinámicas de movilidad intergeneracional—, los llevados a cabo por Kessler y Espinoza (2003) — que arrojan luz sobre las dinámicas estructurales ambiguas o contradictorias de las últimas décadas en Argenti$\mathrm{na}^{7}$ — y la propuesta de Fachelli (2012) para analizar la desigualdad social en este país, desde un enfoque multidimensional.

En Argentina, sumado a la cuestión de definir qué son las clases desde el punto de vista teórico, en los análisis empíricos aparece la dificultad manifiesta de que "el uso

${ }^{5}$ Estas clases medias serían explotadas en términos de propiedad de los medios de producción, pero a su vez serían explotadoras en relación a sus credenciales y cualificación (Wacquant, 1991).

${ }^{6}$ Los nuevos acercamientos a la estratificación y la movilidad social, por su parte, celebran el "giro cultural" en la sociología de las clases sociales, y revalorizan las aportaciones que desde la teoría de Bourdieu pueden hacerse al análisis de esas clases (Devine y Savage, 2005; Vester, 2005; Devine, 2005; Weininger, 2005).

${ }^{7}$ En Jiménez Zunino (2011b) puede verse una explicitación mayor de todos estos aspectos. Aquí la autora, tomando como eje "las dinámicas complejas en las que se insertan los procesos de estratificación de las sociedades contemporáneas" (2011b: 49), hace una muy interesante sistematización y crítica de los estudios clásicos sobre clase, estratificación y movilidad social, para centrarse especialmente en las clases medias y proponer el análisis de los procesos de movilidad social descendente en términos de "desclasamiento" y no de "empobrecimiento", asumiendo la misma perspectiva relacional e histórica. 
del concepto de clase social muchas veces se reduce a su posible traducción estadística, que no necesariamente sigue los criterios que se postula en el nivel conceptual" (Del Cueto y Luzzi, 2008: 13). Así, desde el pionero estudio de Gino Germani (1955) se hacía visible este problema. Y esta dificultad obliga a tomar una serie de decisiones teóricas, metodológicas y técnicas a fin de articular lo más adecuadamente posible el enfoque teórico con la disponibilidad de la información empírica.

En la perspectiva teórica en que ubicamos nuestro trabajo, las clases sociales son, en primer lugar, construcciones operadas por el investigador (son "clases en el papel", en el sentido de Bourdieu, 1990). Se definen por la distribución desigual de los distintos recursos sociales, y, más concretamente, a partir del volumen y estructura del capital (económico, cultural, social y simbólico) ${ }^{8}$ y de su trayectoria, considerados en términos relacionales (Bourdieu, op. cit.). Por otra parte, en la constitución de esas clases, no sólo es necesario considerar las relaciones objetivas identificables en un espacio social concreto, sino que también es fundamental dar cuenta de las relaciones simbólicas que ellas mantienen entre sí, duplicando de ese modo, la disponibilidad diferencial de los recursos y con ello, las relaciones de fuerza y de lucha.

Analizar entonces la dinámica de la reproducción social, supone, en primer lugar, captar su "sentido objetivo" a través de la construcción del espacio pluridimensional de posiciones donde se insertan las distintas clases de agentes (en el sentido estadístico y como una estructura de relaciones objetivas) y, en segundo lugar, dar cuenta de los "sentidos vividos" (Bourdieu, 1991) y de las prácticas concretas9 que esos agentes ponen en marcha (Gutiérrez, 2011).

De esta manera, consideramos el "espacio social" como una construcción teórica que, tomando simultáneamente un conjunto de variables relativas a recursos económicos y culturales y apelando a métodos específicos, nos permite dar cuenta de la estructura de las relaciones de desigualdad, caracterizar las diferentes posiciones de nuestras unidades de análisis, e identificar clases y fracciones de clase.

Aquí se encuentra implícita una perspectiva ontológica que hace desaparecer el problema de la existencia o no de clases, proponiendo la existencia real del espacio de

${ }^{8}$ La propuesta bourdieusiana es que, en las sociedades capitalistas, los dos recursos fundamentales que estructuran el espacio social son el capital económico y el capital cultural, mientras que los otros dos (capital simbólico y capital social) serían una suerte de sobreañadido que pueden potenciar la eficacia de los más importantes. Su valor concreto en el juego de inversiones y reconversiones de capitales es susceptible de ser visualizado en el análisis de problemáticas específicas, como en el caso de las estrategias escolares de la pequeña burguesía o de los sectores dominantes franceses que analiza Bourdieu (1988 y 2013) o incluso en la definición de diferentes modos de reproducción social en la pobreza (Gutiérrez, 2004).

${ }^{9}$ Un estudio detallado sobre la dialéctica entre las posiciones y las tomas de posición culturales, tomando como referente empírico la ciudad de Villa María (Córdoba, Argentina), que implica también un desarrollo sobre el modo como se inserta esta perspectiva en el campo de los estudios de comunicación, puede verse en Mansilla (2011). 
relaciones, sin renunciar a la idea de clase como diferenciación social de los agentes que ocupan distintas posiciones en aquel espacio. No se trata entonces de dar cuenta de clases sociales definidas previamente, sino de reconstruir la estructura del espacio social para identificar en él a conjuntos de agentes con posiciones semejantes.

Finalmente, señalemos que como espacio virtual, la noción de espacio social no se confunde con la de espacio geográfico: define proximidades y distancias sociales, y por ello es diferente al espacio físico y su construcción no supone una dimensión territorial (Bourdieu 1990, 1997). Sin embargo, en el análisis de problemáticas concretas se puede observar cómo ambos espacios se relacionan, y visualizar las huellas que en el espacio físico dejan las desigualdades sociales (con barrios ricos y barriadas pobres, por ejemplo), en la medida en que los modos de apropiación del espacio geográfico dependen de las posibilidades que definen las posiciones en la estructura de relaciones objetivas (Bourdieu, 1999).

Nuestra perspectiva teórica conlleva una metodología y una instrumentación técnica pertinente para el estudio empírico. Para construir el espacio social cordobés utilizamos el análisis multidimensional de datos desarrollado por la escuela francesa de Analyse des données, en particular el análisis de correspondencias múltiples (ACM) y los métodos de clasificación, especialmente el de clasificación jerárquica ascendente (CJA $)^{10}$.

Así, componer la estructura del espacio social cordobés consiste, en un primer momento, en hacer actuar simultáneamente — a través de un ACM - un conjunto de variables activas e identificar sus múltiples relaciones. En un segundo momento, con la aplicación de métodos de clasificación, podremos distinguir diferentes clases sociales — sobre la base de las clases estadísticas diferenciadas por estos métodos- e identificar las relaciones que existen entre las posiciones próximas de nuestras unidades de análisis y las propiedades que las caracterizan.

\subsection{Técnicas y fuentes: su adecuación a la perspectiva teórico-metodológica}

Como información de base recurrimos a una de las fuentes de nuestro Sistema Estadístico Nacional, la Encuesta Permanente de Hogares (EPH) para el Gran Córdoba, en el

${ }^{10}$ Desde nuestra perspectiva es imprescindible la articulación de ambos métodos. En efecto, es necesario en un primer momento la implementación de un análisis de las correspondencias entre las variables, para dar cuenta de la estructura de relaciones donde cada modalidad adquiere su verdadero valor (Bourdieu, 1988). Una vez obtenidos los factores que expresan las relaciones entre las variables, es posible asignar a cada unidad de análisis las coordenadas de su ubicación en el espacio pluridimensional. La clasificación jerárquica ascendente nos permite agrupar las unidades de análisis en base a la medición de las distancias en el espacio construido por el análisis de correspondencias (Bénzecri et al., 1973; Lébart, 1989; Baranger, 1999; Moscoloni, 2005). 
tercer trimestre de los años 2003 y $2011^{11}$, y utilizamos un software específico (SPAD 5.0 de DECISIA).

La EPH captura información sobre viviendas, hogares e individuos. El "hogar" se define a partir de los criterios de corresidencia de sus miembros e implicación común en los gastos de reproducción (vivir bajo un mismo techo y poseer una estructura de gastos compartida). Además, como en la recolección de datos individuales se capturan las relaciones de parentesco de los miembros con relación al jefe de hogar, en la etapa de análisis se pueden recomponer diferentes núcleos familiares y relaciones de parentesco constitutivas del hogar (Torrado, 1998).

Por otra parte, distinguimos las características del hogar que serían seleccionadas como indicadores de sus recursos colectivos, de aquellas características individuales que también conforman los recursos de la familia. Si bien las primeras no presentan mayores inconvenientes, las últimas obligan a considerar algunas alternativas que validen el paso de lo individual a lo colectivo. Estas operaciones implican, entre otras, la utilización de algoritmos matemáticos para convertir características individuales en propiedades colectivas (como, por ejemplo, el ingreso per cápita del hogar o su clima educacional), o bien el establecimiento de un proceso de selección de un referente dentro del hogar $(\mathrm{RH})$. Se trata de elegir a una persona de referencia que, por tener ciertas características, permita asignar sus recursos individuales al grupo ${ }^{12}$.

Así, si las características socio-ocupacionales de los miembros del hogar implican capitales de diferentes especies, como la calificación y la jerarquía ocupacional, éstos deben entrar en la conformación del volumen y estructura patrimonial colectiva. Sin embargo, debemos contemplar que la capacidad de esos recursos para jugar como capitales de la familia se encuentra mediada por la posición relativa del miembro que lo aporta.

La lectura de antecedentes sobre la problemática parece indicar que la elección del "jefe de hogar" como la persona de referencia es el criterio más adecuado. No obstante, una rápida revisión de esta condición deja ver cierta indeterminación en su uso. Al ser los propios miembros del hogar los que identifican a un jefe, se carece de un criterio unificado: suele ser reconocido como tal quien posee mayor edad, o aquel que se ocupa de las tareas del hogar o el miembro que realiza el principal aporte económico. De

${ }^{11}$ La EPH es un programa nacional de captura sistemática y permanente de datos sobre las características demográficas y socioeconómicas fundamentales de la población, vinculadas a la fuerza de trabajo. Se realiza trimestralmente, tiene cobertura nacional y abarca los mayores centros urbanos del país. Elegimos el tercer trimestre por la posibilidad de relacionar nuestros resultados con otros relevamientos continuos que se realizan en ese mismo período: la Encuesta Anual de Hogares Urbanos (EAHU) y la Encuesta Nacional sobre Acceso y Uso de de Tecnologías de la Información y Comunicación (ENTIC).

12 De ambos procedimientos, tal vez el que más definiciones exija sea el de seleccionar un referente dentro del hogar (RH), en la medida en que se debe contemplar no sólo la naturaleza de los recursos que el referente transfiere al grupo, sino también el lugar que él ocupa en el sistema de relaciones de parentesco y de poder. 
este modo, la idea de jefatura de hogar, convertida en categoría central de la encuesta, responde a las diversas definiciones que el sentido común le asigna e impide tomarla como única condición para establecer un referente.

Para nuestro trabajo definimos un conjunto de criterios de selección de ese referente, que tuvieron como objetivo fundamental recuperar la trayectoria de clase del grupo familiar, por lo que en una primera instancia contemplamos el número de generaciones presentes en cada hogar. Para su determinación consideramos las relaciones de filiación y parentesco a partir de quien era reconocido como jefe, y luego definimos una combinatoria de reglas de selección de los posibles referentes, que tomó de manera relacional la edad, filiación y pertenencia generacional de todos los miembros del hogar.

Una vez identificados los miembros que podían ocupar ese lugar de referencia, procedimos a aplicar una serie de criterios de selección jerárquicos y excluyentes. Esto implicó establecer cuáles debían ser los recursos individuales a considerar, fundamentalmente aquellos vinculados a los capitales económico y cultural de las personas seleccionadas. Asimismo se evaluó su peso relativo en el sistema de relaciones familiares, lo que finalmente permitió la selección final de un único referente para cada hogar. En resumen, se identificó aquel miembro del grupo que "tiene la mayor responsabilidad en el mantenimiento del hogar o que ejerce la mayor influencia en las decisiones concernientes al consumo" (Torrado, 1998: 132).

Conforme a estas definiciones sobre nuestra unidad de análisis y la información disponible en la EPH, se procedió a la realización de un análisis de las correspondencias sobre un conjunto de indicadores de los recursos de los hogares a fin de obtener las estructuras inmanentes que definen los condicionamientos asociados a las diferentes posiciones sociales. Este trabajo implicó la selección de un conjunto de variables activas correspondientes al hogar y su referente, al mismo tiempo que las demás propiedades quedaron como variables ilustrativas o suplementarias. Tal tarea de selección involucró la revisión de criterios aplicados en otros trabajos y la realización de diferentes pruebas para llegar a establecer aquellos indicadores que, dentro de las limitaciones presentes en los datos recogidos por la EPH, condensaran la estructura patrimonial de los hogares y permitieran una lectura lo más clara posible de las relaciones de desigualdad no sólo para un trimestre particular, sino también para analizar su trayectoria a lo largo de los últimos diez años. ${ }^{13}$

Entre los trabajos que se propusieron realizar una medición empírica de las clases sociales a partir de las fuentes del sistema estadístico nacional argentino, se destaca el de Susana Torrado (1998). Se propone la construcción de un nomenclador de la condición socio-ocupacional en el que los estratos se definen a partir de seis variables

${ }^{13}$ Una primera aproximación a la construcción del espacio social cordobés puede verse en Gutiérrez y Mansilla, 2013. 
de naturaleza económica: condición, sector y rama de actividad, grupo y categoría de ocupación y tamaño del establecimiento.

Desde nuestra perspectiva, es necesario incorporar también indicadores que remitan a la otra especie de capital que estructura el espacio social. El capital cultural es captado por la EPH en su forma legitimada, es decir como capital escolar, por lo que incorporamos el máximo nivel de educación alcanzado por el referente como una variable activa ${ }^{14}$. Además, el capital cultural es una dimensión que está presente en otras variables activas que incorporamos, tales como la jerarquía y la calificación ocupacional ${ }^{15}$.

En suma, para el análisis de las correspondencias optamos por seleccionar un conjunto de ocho variables activas. En relación con la disponibilidad de recursos económicos del hogar tomamos en cuenta el "ingreso per cápita familiar" (IPCF, considerado en deciles del Aglomerado). A su vez, seleccionamos ciertas características de su referente, tales como sexo, edad, situación conyugal, nivel educativo, otras vinculadas a su inserción en las relaciones de producción —en particular, su jerarquía y calificación ocupacional- $\mathrm{y}$, finalmente, su ingreso total individual ${ }^{16}$. La consideración de estas últimas características nos obligó a filtrar aquellos hogares donde el referente fuese "in-

${ }^{14} \mathrm{Al}$ incorporar también como variable activa la edad del referente, intentamos relativizar el valor de la educación como recurso, y de alguna manera tener en cuenta el posible efecto de devaluación de los títulos por la dinámica de los modos de reproducción que utilizan el mercado escolar. Por la misma razón no utilizamos variables que toman como indicador el promedio de los años de escolaridad de los miembros del hogar (Torrado, 1998; Fachelli, 2012), ya que así se elimina la jerarquía de las titulaciones y la dimensión histórica de su valor.

${ }^{15}$ En el sistema estadístico argentino la calificación alude al grado de complejidad de las tareas desarrolladas en una ocupación. Si bien esta calificación no se refiere en modo alguno al nivel educativo de las personas implica un componente cultural en su naturaleza que se encuentra asociado por lo general a una mayor retribución económica. Sus modalidades en orden decreciente van de calificación profesional a técnica, luego operativa y por último las no calificadas. Por su parte la jerarquía ocupacional refiere a la existencia de formas jerárquico-organizativas de los procesos de trabajo y se establece a partir de la existencia de líneas de mando que hacen visible la posición de cada ocupación en el ordenamiento interno de las unidades productivas. Implica entonces a diferentes especies de capital y consta de cuatro categorías: dirección, jefatura y ejecución directa —estas tres ordenadas en función del nivel jerárquico-y ocupaciones independientes, denominadas así ya que se encuentran fuera de las relaciones jerárquicas por no trabajar en relación de dependencia ni tener personal a cargo.

${ }^{16}$ A diferencia de otros estudios decidimos dejar como variables ilustrativas tanto al sector y la rama de actividad como al tamaño del establecimiento dado que estas propiedades no necesariamente expresan capitales o poderes en el marco del espacio social general a construir, lo que fue confirmado en diferentes ensayos previos. 
activo", ya que la EPH no captura esta información para esos casos: en consecuencia, trabajamos con un total de 532 sobre los 682 hogares de la muestra ${ }^{17}$.

\subsection{La estructura del espacio social cordobés en 2011}

En el Diagrama 1 mostramos el espacio social del Gran Córdoba para el año 2011'8, construido por 51 modalidades activas y representado en sus dos primeras dimensiones; es decir, en los dos primeros factores, que expresan el $13,14 \%$ de la inercia total ${ }^{19}$.

El primer factor (representado en sentido vertical) opone las familias mejor provistas en volumen global de capital a aquellas con una menor provisión de recursos. Expresando el 7,2\% de la inercia total y conformado principalmente por las contribuciones del IPCF $(23,9)^{20}$, la calificación ocupacional del referente de hogar $(20,8) \mathrm{su}$ ingreso total $(20,6)$ y su nivel de instrucción $(19,3)$, este eje diferencia en la región superior del plano a las posiciones sociales que tienen un mayor volumen global de capital: un máximo de recursos económicos — expresado en la pertenencia al $10^{\circ}$ decil en el IPCF - y un alto volumen de recursos culturales — indicado por el nivel de instrucción formal alcanzado por el referente-, que llega a estudios universitarios completos. A estas propiedades se suman otros indicadores del volumen patrimonial, como la calificación y la jerarquía de la ocupación laboral (profesional y directivo).

En la región inferior del espacio, se ubican aquellas posiciones que presentan un menor volumen global de recursos, tanto económicos como culturales. La participación en el primer decil del IPCF y estudios primarios incompletos como máximo nivel de instrucción formal alcanzado, son algunos de los indicadores que expresan más claramente las diferencias que construyen el primer factor. La proyección del resto de las modalidades de las variables activas, en particular aquellas que resultaron de mayor contribución, permite visualizar las principales propiedades que caracterizan esta región inferior del espacio: estudios primarios completos, ausencia de calificación laboral, cuentapropismo e IPCF ubicado en el segundo decil.

${ }^{17}$ Los hogares con un referente "inactivo" y el resto de las variables de la EPH fueron incorporados al análisis en carácter de individuos suplementarios y propiedades ilustrativas respectivamente.

${ }^{18}$ En los diagramas, las modalidades activas se identifican con un rombo; los baricentros de clases con un círculo negro, las modalidades suplementarias fueron identificadas con un cuadrado y junto a las fracciones de clase están representadas en color gris y cursiva.

${ }^{19}$ Se entiende por inercia total de la nube de puntos a la medida de dispersión de los perfiles que, como indicador de esta dispersión, permite medir la relación entre las variables.

${ }^{20}$ Para la caracterización de los factores el número entre paréntesis expresa la contribución acumulada de la variable a su conformación. 
El segundo factor (representado en sentido horizontal) distingue las regiones medias del espacio social. La conformación de este factor desplaza hacia la izquierda a aquellas posiciones que disponen de un volumen global medio de capital, diferenciándolas de la región derecha del espacio, donde el primer factor opone los extremos (alto y bajo) en el sentido vertical del diagrama.

A su vez, estas posiciones medias del espacio social se diferencian entre sí por el volumen global de capital poseído. Es decir, podemos realizar una lectura que coloca en relieve los condicionamientos asociados a los cuadrantes superior e inferior de la región izquierda del espacio, conformando dos zonas con claras diferencias de propiedades respecto al volumen patrimonial de las familias. El cuadrante superior muestra ingresos familiares medios altos, asociados a un IPCF que va del $7^{\circ}$ al $9^{\circ}$ decil, junto a estudios que van del nivel secundario completo al superior universitario incompleto y calificación laboral técnica de los referentes de hogar. Por su parte, el cuadrante inferior izquierdo comprende ingresos del orden del $2^{\circ}$ y $4^{\circ}$ decil, sumados a estudios secundarios incompletos y calificación laboral operativa, como propiedades del referente.

Diagrama 1: El espacio social Cordobés 2011en el Plano De los EJes 1 y 2

(13,14\% DE INERCIA Y 51 MODALIDADES ACTIVAS)

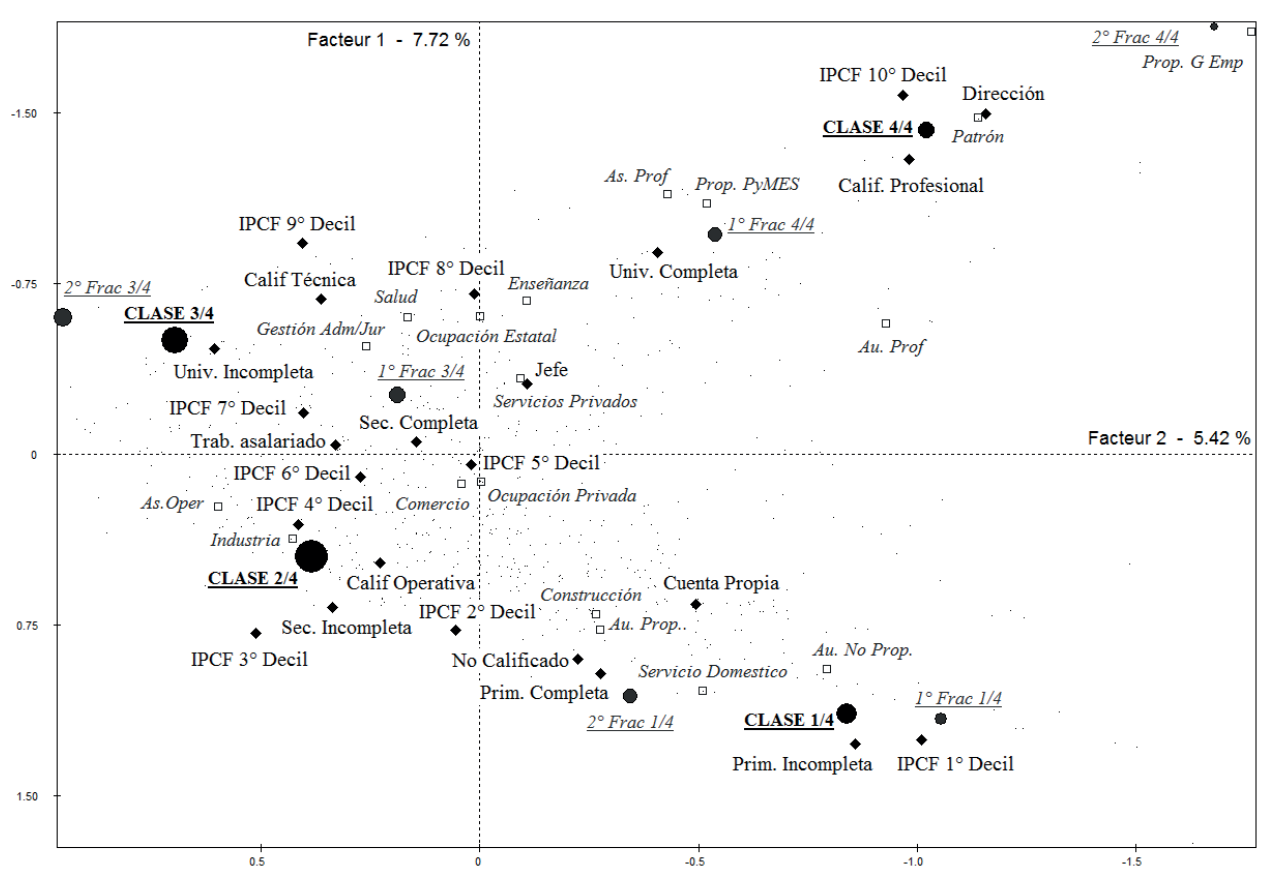

Fuente: Elaboración propia, sobre la base de la EPH, tercer trimestre de 2011. 
El elevado número de familias que se posicionan aquí, junto a los límites difusos entre ambas regiones del espacio, hace que las diferencias expresadas por el segundo factor no sean de simple lectura. Y aquí es necesario precisar algunas cuestiones ligadas a los métodos utilizados. Por un lado, debemos reconocer que la necesidad de resumir visualmente a sus dos primeras dimensiones la naturaleza multidimensional del espacio social, nos dificulta presentar a simple vista las diferencias en la estructura patrimonial completa. Pero, por otro, tenemos que subrayar que esta limitación visual no nos impide dar cuenta analíticamente de la estructuración del capital: por ello, seguidamente, volveremos sobre la multiplicidad de coordenadas factoriales que definen cada posición, para formar diferentes "clases de familias" en tanto posiciones próximas en aquel espacio social original (multidimensional), a través de la aplicación de algoritmos de clasificación.

\subsection{Las clases estadísticas o "clases en el papel"}

La aplicación de la clasificación jerárquica ascendente (CJA) tomó como base los primeros cuatro factores o dimensiones del espacio social original. Como puede observarse en la Tabla 1, la inercia total del ACM realizado se descompone en 43 ejes factoriales con 22 de ellos por encima del valor propio medio. Sin embargo, sólo los cuatro primeros ejes presentan un decrecimiento irregular de la inercia, y a partir del quinto se muestra una cierta regularidad de decrecimiento: la nube exhibe cuatro direcciones de alargamiento principales que estarían expresando claras diferencias entre las familias, mientras que los demás ejes responderían a diferencias más específicas sobre aspectos puntuales que las harían de difícil interpretación. En consecuencia, para la realización de la CJA optamos por considerar los cuatro primero factores con una acumulación de inercia del 22,69\%.

De ello resultó la construcción de un dendograma que mostró un corte óptimo para la composición de cuatro grandes clases (y seis fracciones) en correspondencia directa con las regiones antes descriptas.

Como puede observarse, la partición inicial muestra cuatro clases compuestas por un $20 \%, 35 \%, 30 \%$ y $17 \%$ respectivamente. A su vez permite suponer, al interior de las cuatro grandes clases constituidas por las principales diferencias, la existencia de grupos o fracciones conformados por diferencias secundarias. Así, una segunda partición posibilita explorar el número y composición de cada una de ellas. Las clases y fracciones que resultaron de interés se encuentran proyectadas en el Diagrama 1, a partir de la ubicación del baricentro de la nube que conforma cada agrupamiento.

La descripción de las grandes regiones del espacio social cordobés realizada sobre el Diagrama 1 nos permitió observar que las principales diferencias que lo estructuran fueron definidas por las posiciones de las clases que se ubican en extremos opuestos del primer factor. En consecuencia, podemos avanzar en la descripción del espacio social y detallar las características asociadas a estas posiciones, incorporando, junto a las variables activas que estructuran el espacio, otras características que jugaron como variables suplementarias y que resultaron asociadas significativamente a cada clase. 
Tabla 1: Valores propios e histograma para el Espacio Social Córdoba 2011 ( $3^{\circ}$ TRIMESTRE). TRAZA DE LA MATRIZ DE InERCiA: 4,6275

\begin{tabular}{|c|c|c|c|c|}
\hline $\mathrm{N}^{\mathrm{o}}$ & $\begin{array}{l}\text { Valor } \\
\text { propio }\end{array}$ & $\begin{array}{c}\text { Inercia } \\
(\%)\end{array}$ & $\begin{array}{c}\text { Inercia } \\
(\% \\
\text { acum })\end{array}$ & Histograma de valores propios \\
\hline 1 & 0,357272 & 7,72 & 7,72 & \\
\hline 2 & 0,2508 & 5,42 & 13,14 & \\
\hline 3 & 0,2367 & 5,12 & 18,25 & \\
\hline 4 & 0,2054 & 4,44 & 22,69 & \\
\hline 5 & 0,1775 & 3,84 & 26,53 & \\
\hline 6 & 0,1745 & 3,77 & 30,30 & \\
\hline 7 & 0,1671 & 3,61 & 33,91 & \\
\hline 8 & 0,1649 & 3,56 & 37,47 & \\
\hline 9 & 0,1525 & 3,30 & 40,77 & \\
\hline 10 & 0,1476 & 3,19 & 43,96 & \\
\hline 11 & 0,1460 & 3,15 & 47,11 & \\
\hline 12 & 0,1425 & 3,08 & 50,19 & \\
\hline 13 & 0,1340 & 2,89 & 53,09 & \\
\hline 14 & 0,1337 & 2,89 & 55,98 & \\
\hline 15 & 0,1305 & 2,82 & 58,80 & \\
\hline --- & $\ldots$ & $\ldots$ & $\ldots$ & --- \\
\hline 35 & 0,0438 & 0,95 & 98,77 & \\
\hline 36 & 0,0332 & 0,72 & 99,49 & \\
\hline 37 & 0,0216 & 0,47 & 99,95 & \\
\hline 38 & 0,0017 & 0,04 & 99,99 & \\
\hline 39 & 0,0005 & 0,01 & 100,00 & \\
\hline 40 & 0,0000 & 0,00 & 100,00 & \\
\hline 41 & 0,0000 & 0,00 & 100,00 & \\
\hline 42 & 0,0000 & 0,00 & 100,00 & \\
\hline 43 & 0,0000 & 0,00 & 100,00 & \\
\hline
\end{tabular}

Fuente: Elaboración propia sobre la base de la EPH, tercer trimestre de 2011. 
Gráfico 1: Espacio Social Córdoba 2011 - Dendograma de la CJA para los Primeros 4 Factores (22,69\% DE inercia). Particiones para 4 Clases y 6 Fracciones.

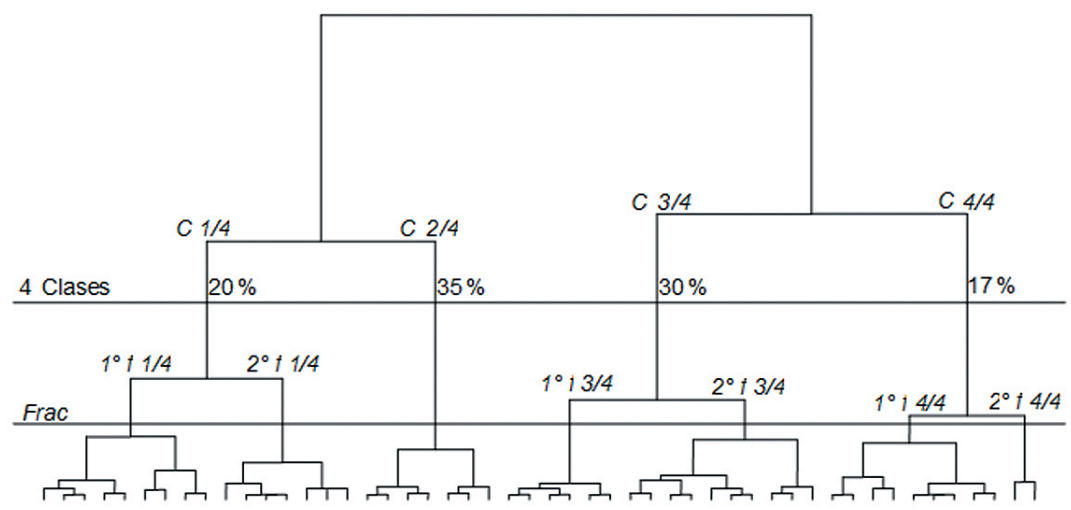

Fuente: Elaboración propia sobre la base de la EPH, tercer trimestre de 2011.

\subsubsection{Los extremos del espacio social}

\subsubsection{La región de bajo volumen de capital}

Si el Diagrama 1 muestra la distribución de las familias sobre el primer factor, diferenciando posiciones según el volumen global de sus recursos, la clasificación jerárquica ascendente permite recortar una primera clase de un $20 \%$ (clase 1/4) que se diferencia del resto por su bajo volumen de recursos. La lectura de las características asociadas a este grupo, presentadas en la Tabla 2, permiten denominar al conjunto de familias que lo conforman como clase de bajos recursos o clase dominada.

Estas familias se caracterizan por su bajo volumen global de capital, con una estructura patrimonial asociada a bajos ingresos: IPCF, ingreso total del referente, su ingreso por ocupación principal y el ingreso total familiar en el $1^{\circ}$ decil. A su vez se asocian, en lo que respecta a la ocupación del referente, a la ausencia de calificación laboral, al servicio doméstico, al cuentapropismo y a la construcción. Estos poseen cierta precariedad en el trabajo: escasa antigüedad laboral, en pequeños establecimientos del ámbito privado, o se desempeñan como trabajadores autónomos, y no poseen cobertura médica. El capital escolar va de nivel primario incompleto a primario completo. Respecto a las características de los hogares de esta clase, puede observarse una asociación significativa con referentes femeninos de edad mayor. Con relación a las viviendas, se observan asociaciones significativas con problemas de hacinamiento y condición de ocupante como régimen de tenencia. Por último, puede señalarse que esta clase de familias se asocia a la recepción de subsidios y ayuda material. 
Tabla 2: Principales Características asociadas a la Clase $1 / 4$ $\left(3^{\circ} \text { TRIMESTRE DE } 2011\right)^{21}$

CLASE 1/4: CLASE BAJA DOMINADA (20\%) Bajo volumen global de capital con una estructura patrimonial asociada a bajas calificaciones laborales, capital escolar de nivel primario e IPCF entre el $1^{\circ}$ y $2^{\circ}$ decil.

Nombre de la variable Modalidad asociada Valor-Test

Ingreso Per Cápita Familiar

$1^{\circ}$ Decil 10,92

Ingreso Total del RH

$1^{\circ}$ Decil 9,93

Ingreso Ocupación Principal del RH

$1^{\circ}$ Decil 9,80

Ingreso Total Familiar

$1^{\circ}$ Decil 9,75

Calificación Ocupacional del RH

No Calificado 9,56

Tipo de cobertura médica RH

No posee

Nivel educativo del RH

Primaria Incompleta

Recepción de subsidio o ayuda social (en dinero)

Sí

Rama de actividad del RH

Servicio Doméstico

Nivel educativo del RH

Primaria Completa

Tamaño del establecimiento del RH

Hasta 5 Personas

Categoría de Actividad del RH

Cuenta Propia

Condición Socio Ocupacional del RH

Au. No Propietario

Sexo del RH

Mujer

Edad del RH

50 a 64 años

Ámbito laboral del RH

Ocupación Privada

Régimen de Tenencia de la Vivienda

Ocupante

Cantidad de miembros por ambiente exclusivo

Más de 3 personas

Rama de actividad del RH

Construcción

Menos de un mes

Fuente: Elaboración propia sobre la base de la EPH, tercer trimestre de 2011.

${ }^{21}$ Cada modalidad asociada a la clase se acompaña con su valor-test. "El mismo mide el desvío entre la proporción en la clase y la proporción general en número de desvíos estándar de una ley normal. El valor test para una modalidad de una variable nominal es entonces un criterio estadístico asociado a la comparación de los efectivos en el marco de una ley hipergeométrica" (Moscoloni, 2005: 188) Utilizaremos los valores test más importantes (siempre mayores a 2) para exponer los elementos más característicos de cada clase ordenados según el grado en que se encuentren asociados. 
Ahora bien, si el bajo volumen de recursos caracteriza a esta clase, existen al interior del grupo diferencias secundarias que permiten distinguir dos fracciones con distintas estructuras patrimoniales: $1^{\circ}$ Frac. $1 / 4$ y $2^{\circ}$ Frac. $1 / 4$ (ver Gráfico 1 y su proyección en el Diagrama 1). Si bien ambas fracciones poseen un bajo volumen global de capital expresado en ingresos dentro de los primeros deciles y un referente de hogar que no supera los estudios de nivel primario, estos grupos se muestran diferentes en torno al sexo del referente y el tipo de inserción laboral que éste logra. Así, los factores constitutivos de la clase se encuentran — en cada fracción - mediados por las determinaciones que impone el género. (Ver Tabla 3)

\section{TABla 3: Principales CARACTERÍSTiCAS ASOCIADAS A LAS FRACCIONES DE LA Clase BAJA DOMinAda ( $3^{\circ}$ TRIMESTRE DE 2011)}

\begin{tabular}{|c|c|c|}
\hline & $1^{\circ}$ fracción baja dominada $(10 \%)$ & $2^{\circ}$ fracción baja dominada $(12 \%)$ \\
\hline Variables & Modalidad asociada (Valor-Test) & Modalidad asociada (Valor-Test) \\
\hline Sexo del referente & Mujer $(6,64)$ & Varón $(3,15)$ \\
\hline Rama de actividad & Servicio Doméstico $(7,80)$ & Construcción $(7,34)$ \\
\hline Calificación Ocupacional & No Calificado $(11,29)$ & Operativa $(6,62)$ \\
\hline Ingreso Per Cápita & $1^{\circ} \operatorname{Decil}(7,59)$ & $1^{\circ} \operatorname{Decil}(6,62)$ \\
\hline Nivel educativo & Primaria Completa $(4,56)$ & Primaria Incompleta $(5,23)$ \\
\hline Cobertura médica & No paga ni le descuentan $(5,43)$ & \\
\hline Situación conyugal & Divorciado $(4,67)$ - Viudo $(2,48)$ & - \\
\hline Categoría de Actividad & & Cuenta Propia $(5,74)$ \\
\hline Miembros del hogar & - & 6 personas o más $(2,44)$ \\
\hline
\end{tabular}

Fuente: Elaboración propia sobre la base de la EPH, tercer trimestre de 2011.

La clase está compuesta por una primera fracción de un $10 \%$, que agrupa familias que poseen en un alto porcentaje (casi el $80 \%$ ) un referente femenino. Se trata de mujeres divorciadas o viudas, sin calificación laboral y que en su mayoría se desempeñan en servicio doméstico.

Completa la clase baja una segunda fracción de aproximadamente un $12 \%$ de hogares que en su mayoría poseen un referente masculino, vinculado a la construcción y al cuentapropismo, con calificación laboral operativa, y que conforman hogares numerosos. 
2.4.1.2. La región de alto volumen de capital

Tabla 4: Principales características asociadas Clase alta dominante ( $3^{\circ}$ TRIMESTRE DE 2011)

CLASE 4/4: CLASE ALTA DOMINANTE (17\%). Alto volumen global de capital con una estructura patrimonial asociada a la propiedad de empresas o el control de la fuerza laboral, calificaciones laborales profesionales e Ingresos en el $10^{\circ}$ decil

Nombre de la variable

Ingreso Total del RH

Calificación Ocupacional del RH

Ingreso Ocupación Principal del RH

Ingreso Per Cápita Familiar

Carácter Ocupacional del RH

Jerarquía Ocupacional del RH

Ingreso Total Familiar

Nivel educativo del RH

Categoría de Actividad del RH

Condición Socio Ocupacional del RH

Cantidad de ambientes/habitaciones de la vivienda

Ámbito laboral del RH

Rama de actividad del RH

Condición Socio Ocupacional del RH

Sexo del RH

\begin{tabular}{lc} 
Modalidad asociada & Valor-Test \\
$10^{\circ}$ Decil & 14,12 \\
Profesional & 12,51 \\
$10^{\circ}$ Decil & 12,17 \\
$10^{\circ}$ Decil & 9,96 \\
Directivos & 9,00 \\
Dirección & 9,00 \\
$10^{\circ}$ Decil & 8,67 \\
Univ. Completa & 8,46 \\
Patrón & 8,37 \\
Prop. G Emp & 8,36 \\
Cinco o más & 4,88 \\
Estatal & 3,72 \\
Enseñanza & 3,47 \\
Prop. Pymes & 3,34 \\
Varón & 2,51 \\
\hline
\end{tabular}

Fuente: Elaboración propia sobre la base de la EPH, tercer trimestre de 2011.

Muy diferente es la situación de las familias que se ubican en la parte superior del espacio. Constituidas por un $17 \%$, conforman la clase $4 / 4$, que puede denominarse clase alta dominante (ver Tabla 4). Con un alto volumen global de capital, su estructura patrimonial se encuentra asociada a ingresos que se ubican en el $10^{\circ}$ decil. Está constituida por familias que en su mayoría poseen un referente de hogar que ha alcanzado una calificación ocupacional profesional, ocupando puestos directivos. Estos son patrones 
o propietarios tanto de grandes empresas como de pymes y poseen un nivel de instrucción superior universitario. A su vez son en su mayoría referentes de hogar varones, asociados con ocupaciones en el Estado, particularmente en la rama de la educación y sus viviendas poseen buenas condiciones habitacionales.

Tabla 5: Principales Características asociadas a las fracciones DE LA Clase ALTA DOMinANTE ( $3^{\circ}$ TRIMESTRE DE 2011)

\begin{tabular}{|c|c|c|}
\hline & $1^{\circ}$ fracción alta dominate $(13 \%)$ & $2^{\circ}$ fracción alta dominante $(5 \%)$ \\
\hline Variables & Modalidad asociada (Valor-Test) & Modalidad asociada (Valor-Test) \\
\hline Sexo del RH & Varón $(3,23)$ & Varón $(2,38)$ \\
\hline Cond. Socio Ocupacional & Asalariado Profesional $(5,53)$ & Prop. G Emp $(7,64)$ PyMES $(3,23)$ \\
\hline Ingreso Per Cápita & $8^{\circ}$ Decil $(3,68)$ & $10^{\circ}$ Decil $(10,51)$ \\
\hline Nivel educativo & Univ. Completa $(7,29)$ & Univ. Completa $(3,48)$ \\
\hline Rama de actividad & Enseñanza $(3,53)$ & Servicios Privados $(3,46)$ \\
\hline Jerarquía Ocupacional & Dirección $(2,85)$ & Dirección $(8,93)$ - Jefe $(2,67)$ \\
\hline Tecnología Ocupacional & Sistemas y Eq Inf $(3,62)$ & \\
\hline Ámbito laboral & Estatal $(4,57)$ & \\
\hline Categoría de Actividad & - & Patrón $(8,73)$ \\
\hline
\end{tabular}

Fuente: Elaboración propia sobre la base de la EPH, tercer trimestre de 2011.

Por su parte, la fracción más pequeña $\left(2^{\circ}\right.$ frac. $4 / 4$ con un $5 \%$ aproximadamente) presenta fuertes asociaciones con indicadores de propiedad de empresas y referentes patrones, ocupando cargos de dirección o jefatura en la rama de los servicios privados.

\subsubsection{La región media del espacio social}

Con 338 casos efectivos, quienes se posicionan en esta región representan aproximadamente el $64 \%$ del universo, lo que conforma un grupo muy heterogéneo y susceptible de ser reagrupado en dos grandes clases que permiten una mejor descripción de las desigualdades existentes en este vasto sector.

Ubicadas en su mayoría en el cuadrante inferior izquierdo del diagrama, se encuentran posiciones con un volumen global medio-bajo de recursos. Agrupa posiciones a las que hemos denominado clase media dominada y se corresponde con el segundo agrupamiento en la partición inicial del dendograma o clase $2 / 4$. Conformando un $35 \%$ de casos, las familias pertenecientes a esta clase poseen una estructura patrimonial 
asociada principalmente a un IPCF que va del $3^{\circ}$ al $5^{\circ}$ decil. Aunque los ingresos del referente, tanto por su ocupación principal como sus ingresos totales, se ubican en deciles más altos, el elevado número de miembros del hogar tiende a disminuir su IPCF. La clase posee referentes de hogares asociados principalmente a calificaciones laborales operativas, en la industria, y con niveles de instrucción ubicados en los estudios secundarios incompletos. A estas características se suman otras con un menor nivel de asociación, que vinculan a estos referentes con el cuentapropismo y la construcción.

TABla 6: Principales CARaCterísticas asociadas ClaSE MEdia DOMINADA ( $3^{\circ}$ TRIMESTRE DE 2011)

CLASE 2/4: CLASE MEDIA DOMINADA (35\%): Volumen y estructura patrimonial asociados a calificaciones laborales operativas, capital escolar medio incompleto e IPCF entre el $3^{\circ}$ y $5^{\circ}$ decil.

Nombre de la variable

Calificación Ocupacional del RH

Nivel educativo del RH

Ingreso Per Cápita Familiar

Ingreso Per Cápita Familiar

Rama de actividad del RH

Cantidad de miembros del hogar

Ingreso Ocupación Principal del RH

Jerarquía Ocupacional del RH

Rama de actividad del RH

Ingreso Per Cápita Familiar

Ingreso Total del RH
Modalidad asociada Valor-Test

Operativa

12,49

Sec. Incompleta

$3^{\circ}$ Decil

6,97

$4^{\circ}$ Decil

4,92

Industria

4,70

6 personas o más

3,73

$7^{\circ}$ Decil

3,28

Cuenta Propia

3,16

Construcción

2,80

$5^{\circ}$ Decil

2,66

$8^{\circ}$ Decil

2,52

Fuente: Elaboración propia sobre la base de la EPH, tercer trimestre de 2011.

Con un mayor volumen global de recursos, encontramos en el cuadrante superior izquierdo un $29 \%$ de familias que constituyen la clase 3/4. (Ver Tabla 7).

Estas familias se caracterizan por poseer referentes jóvenes que alcanzan, en su mayoría, una calificación ocupacional técnica, estudios superiores universitarios incompletos o completos y un IPCF ubicado entre el $7^{\circ}$ y el $9^{\circ}$ decil, con ocupaciones asociadas a los servicios sociales, en particular a la educación, la gestión administra- 
tiva y la salud. Se trataría de una clase que desarrolla sus estrategias aprovechando a su favor ciertos mecanismos de objetivación de su capital escolar y sus instancias de legitimación en el mercado laboral, razón por la cual la hemos denominado clase media dominante. Otras características vinculadas a esta clase muestran hogares unipersonales o pocos numerosos, con referentes que en su mayoría son mujeres solteras y con hogares sin presencia de menores de diez años.

Tabla 7: Principales Características asociadas Clase 3/4 ( $3^{\circ}$ Trimestre De 2011)

CLASE 3/4: CLASE MEDIA DOMINANTE (29\%): Alto volumen global de capital con una estructura patrimonial asociada a calificaciones laborales técnicas, capital escolar Superior Universitario e IPCF entre el $7^{\circ}$ y $9^{\circ}$ decil.

Nombre de la variable

Edad del RH

Calificación Ocupacional del RH

Situación conyugal del RH

Sexo del RH

Nivel educativo del RH

Ingreso Per Cápita Familiar

Nivel educativo del RH

Carácter Ocupacional del RH

Rama de actividad del RH

Cantidad de miembros del hogar menores de 10 años

Ingreso Per Cápita Familiar

Cantidad de miembros del hogar

Carácter Ocupacional del RH

Carácter Ocupacional del RH

Cantidad de miembros del hogar
Modalidad asociada Valor-Test

Hasta 34 años

10,15

Técnica

9,34

Soltero

8,84

Mujer

6,74

Univ. Incompleta

6,31

$7^{\circ}$ Decil

6,18

Univ. Completa

4,74

Educación

4,67

Servicios Sociales

4,35

Sin menores

4,30

$9^{\circ}$ Decil

4,19

2 personas

3,78

Gestión Adm/Jur

3,74

Salud

3,47

Unipersonal

Fuente: Elaboración propia sobre la base de la EPH, tercer trimestre de 2011.

Si las clases medias dominadas se mostraron homogéneas para una segunda partición (ver Gráfico 1) no sucede lo mismo con las clases medias dominantes de esta región 
del espacio social. Aquí nuevamente puede observarse la incidencia del género y en particular la feminización de la $1^{\circ}$ fracción que compone la clase media dominante $\left(1^{\circ}\right.$ Frac. 3/4). (Ver Tabla 8).

Se trata de un grupo de aproximadamente $14 \%$ de familias que presentan una fuerte asociación con referentes femeninos, de mayor capital cultural que su fracción vecina (valor test de 6,77 en la modalidad universitario completo frente a uno de 7,78 para estudios universitarios incompletos en la $2^{\circ}$ fracción de la clase $3 / 4$ ) y con estrategias laborales basadas en la ocupación de puestos asociados a la educación y la salud, preferentemente en el ámbito estatal ${ }^{22}$.

Tabla 8: Principales características asociadas a las fracciones DE LA CLASE MEDIA DOMINANTE ( $3^{\circ}$ TRIMESTRE DE 2011)

\begin{tabular}{|c|c|c|}
\hline & $1^{\circ}$ fracción media dominante $(14 \%)$ & $2^{\circ}$ fracción media dominante $(18 \%)$ \\
\hline Variables & Modalidad asociada (Valor-Test) & Modalidad asociada (Valor-Test) \\
\hline Ingreso Per Cápita & $7^{\circ} \operatorname{Decil}(7,51)$ & $9^{\circ} \operatorname{Decil}(6,51)$ \\
\hline Nivel educativo & Univ. Completa $(6,77)$ & Univ. Incompleta $(7,78)$ \\
\hline Calificación Ocupacional & Técnica $(6,53)$ & Técnica $(5,30)$ \\
\hline Carácter Ocupacional & Salud $(3,69)$ & Gestión Adm/Jur $(3,06)$ \\
\hline Rama de actividad & Enseñanza $(4,16)$ & \\
\hline Rama de actividad & Servicios Sociales $(3,88)$ & \\
\hline Ámbito laboral & Estatal $(3,04)$ & \\
\hline Sexo del RH & Mujer $(7,65)$ & - \\
\hline Edad del RH & & Hasta 34 años $(12,10)$ \\
\hline Jerarquía Ocupacional & & asalariado $(3,69)$ \\
\hline Situación conyugal & & Soltero $(8,28)$ \\
\hline Tamaño del hogar & $\longrightarrow$ & Hogar unipersonal $(2,61)$ \\
\hline
\end{tabular}

Fuente: Elaboración propia sobre la base de la EPH, tercer trimestre de 2011.

Completa la clase una segunda fracción $\left(2^{\circ}\right.$ frac. $\left.3 / 4\right)$ asociada a referentes de hogares jóvenes, solteros, que conforman hogares unipersonales, con trabajos asalariados, de calificación técnica, en la gestión administrativa y jurídica.

\footnotetext{
${ }^{22}$ Este perfil permite observar la feminización del llamado "brazo izquierdo del Estado" (Bourdieu, 1999).
} 


\subsubsection{La primacía del análisis relacional y la dinámica del espacio social}

Tal vez el mayor obstáculo epistemológico en el estudio de la dinámica de las clases sociales lo represente alguna forma de sustancialismo: por ejemplo, detenerse sólo en la descripción de cada grupo en sí mismo. Si bien tal caracterización puede conformar una etapa del análisis, es necesario, como hemos mostrado más arriba, un momento previo donde se construyan relacionalmente los grupos caracterizados y que valide la descripción de las relaciones más o menos visibles entre ellos. Para evitar el análisis en términos sustancialistas, hay que proceder de manera relacional tanto en la construcción como en la descripción: en este sentido, no tomamos cada grupo de manera aislada sino que reconstruimos el sistema de relaciones a partir del cual los definimos y caracterizamos, desde proximidades y distancias en el espacio social.

Así, es posible ver que las diferencias que se establecen entre las grandes clases sociales, y que a su vez las constituyen como tales, remiten a relaciones de desigualdad en torno a aspectos centrales de la reproducción económica y social de las familias, en particular la inserción laboral de sus referentes, y los recursos que entran en juego en esas prácticas. Podríamos suponer que de ellas dependen fuertemente otras estrategias, como las de fecundidad, las habitacionales y la apelación a diferentes tipos de ayudas y subsidios.

La región del cuadrante inferior derecho del espacio agrupa posiciones ocupadas por familias en las que el referente de hogar vende su fuerza de trabajo, y lo hace a través de un modo de inserción en el mercado laboral que se caracteriza por no requerir calificación alguna, no percibir aportes ni beneficios sociales y por obtener bajas remuneraciones. Estas posiciones poseen muy bajo volumen patrimonial tanto en lo cultural como en lo económico, aunque la característica diferencial que articula su relación con las demás posiciones es la venta de fuerza de trabajo sin calificación y en situaciones de precariedad laboral. Por su parte, las familias que se ubican en las regiones medias dominadas se caracterizan por vender fuerza de trabajo de baja o media calificación. Este aspecto los diferencia tanto de la clase anterior como de las otras clases medias que, ubicadas en mejores posiciones sociales, venden fuerza de trabajo de mayor calificación. Ello se encuentra en relación con los niveles educativos de sus referentes y repercute directamente en los ingresos que perciben.

Es posible que las diferencias entre los cuadrantes superiores (izquierdo y derecho) respondan tanto a la división social del trabajo, vinculada a la propiedad-no propiedad de medios de producción, como a la división técnica en el proceso de trabajo, entre quienes ejecutan tareas y quienes las dirigen y controlan. Mientras los referentes del cuadrante superior izquierdo (clase media dominante) desarrollan una inserción laboral basada en la venta de fuerza de trabajo altamente calificada, vinculada a ocupaciones estatales en ramas de la educación y la salud (fundamentalmente la $1^{\circ}$ fracción $3 / 4$ ), que articula dicha inserción con su capital cultural (capital escolar asociado a formación universitaria completa), los referentes del cuadrante superior derecho presentan 
asociaciones con cargos y funciones directivas. A su vez, éstos son en su mayoría compradores de fuerza de trabajo, a diferencia de los otros, que la venden $\left(2^{\circ}\right.$ fracción $\left.4 / 4\right)$.

Por otra parte, con relación al capital económico expresado por los IPCF y los ingresos individuales del referente, hay que tener en cuenta que, si bien los datos muestran que la clase alta dominante posee ingresos que se ubican en el $10^{\circ}$ decil, este tramo de los ingresos per cápita es muy amplio (oscila entre $\$ 5000$ y $\$ 30000$ —entre 1163 y 6977 dólares-), a diferencia del $9^{\circ}$ decil de la clase media dominante, que implica ingresos que van de $\$ 3300$ a $\$ 4900$ (de 767 a 1140 dólares), lo que puede esconder profundas desigualdades entre ambos grupos y al interior de la clase alta dominante ${ }^{23}$. Así, podemos suponer que los ingresos de los asalariados estatales de la clase media dominante (limitados en sus ingresos) pueden encontrarse muy por debajo de los de las familias con referentes propietarios de empresas o asalariados profesionales directivos en la esfera pública o privada.

Esto nos permite observar la presencia de lo que se denomina una estructura en quiasma. Es decir, una estructura que, a lo largo del factor que expresa el volumen global de capital, diferencia aquellas regiones donde es posible observar cierta primacía del capital cultural sobre el económico (clases y fracciones que articulan sus estrategias de reproducción a partir de la potencialidad de sus recursos culturales y que generalmente se encuentran ubicadas en la región izquierda del espacio) de aquellas otras donde se observa la primacía del capital económico en las inversiones que definen sus estrategias de reproducción (ubicadas sobre la región derecha del espacio social).

\section{Homología estructural y trayectorias}

Para recuperar la dimensión histórica de esta estructura y analizar la trayectoria de las grandes clases sociales con sus cambios en volumen y estructura patrimonial, hemos construido - bajo los mismos criterios- el espacio social correspondiente a la captura de la EPH del $3^{\circ}$ trimestre del año 2003. Comparar ambas estructuras nos permite realizar una aproximación al análisis de las transformaciones del espacio social cordobés.

\subsection{El espacio social cordobés en 2003}

Realizamos un análisis de las correspondencias considerando las mismas variables y procedimientos que fueron implementados para el 2011, tomando las 642 familias que poseen un referente "activo" de la muestra de 815 hogares.

El sistema de relaciones construido muestra claras semejanzas en la composición de sus regiones y los condicionamientos asociados a ellas. El Diagrama 2 ofrece una

${ }^{23}$ Como referencia, consideremos que en ese momento $1 U \$$ A equivalía a 4,30\$ y que el salario mínimo, vital y móvil se ubicaba en los $2.300 \$$ — 535 dólares, aproximadamente- 
representación de las dos primeras dimensiones del espacio, que expresan el 13,26\% de la inercia total. Al igual que para el año 2011, el primer factor se ubica en sentido vertical y ordena a las familias conforme su volumen global de recursos. Así, aquellas menos provistas ocupan las regiones inferiores, en oposición a las de mayor volumen de capital, ubicadas en la región superior; el segundo factor desplaza las clases medias hacia la izquierda del diagrama. Proyectamos también las características asociadas a fin de lograr una rápida caracterización de cada región del espacio.

Diagrama 2: El espacio Social CORdobés 2003 En El Plano DE LOS EJeS 1 y 2 (13,26\% DE INERCIA Y 51 MODALIDADES ACTIVAS)

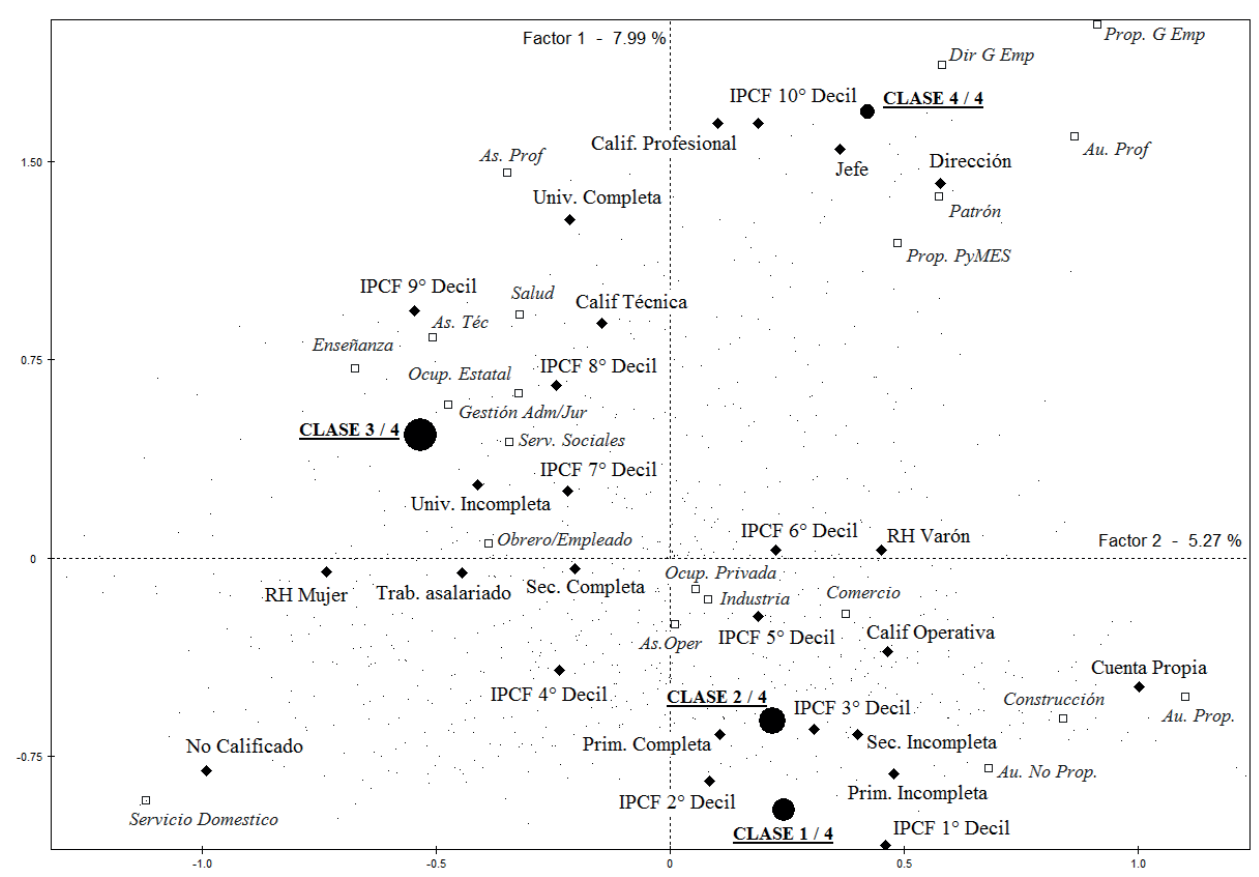

Fuente: Elaboración propia sobre la base de la EPH, tercer trimestre de 2003.

\subsection{Dendograma y caracterización de las clases}

El análisis de la descomposición de la inercia para este año mostró la necesidad de incluir los primeros seis factores para la aplicación de los métodos de clasificación. Como resultado se obtuvo el dendograma del Gráfico 2. El corte óptimo para cuatro clases sociales y la ubicación de los baricentros de cada una (ver Diagrama 2) muestra la 
homología estructural entre el espacio social 2003 y el correspondiente a 2011. Una rápida caracterización de las clases presentes en ambos espacios permitirá dar cuenta de los cambios y continuidades de la estructura social de Córdoba en esta última década.

\section{Gráfico 2: Espacio Social Córdoba 2003 - Dendograma de la CJA PARA LOS PRIMEROS 6 FACTORES $(29,98 \%$ DE INERCIA). Particiones PARA 4 Clases}

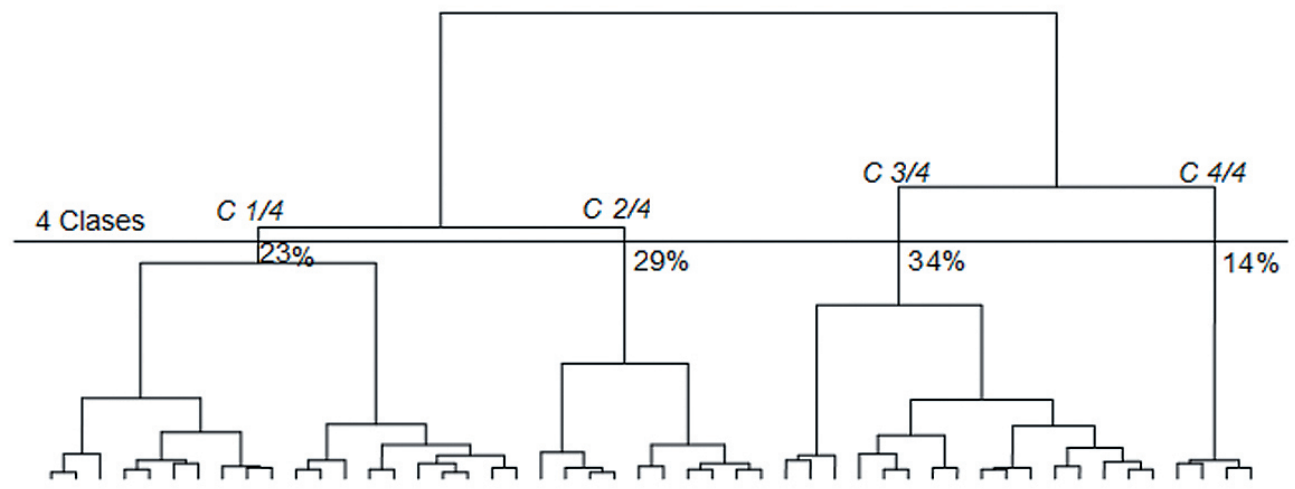

Fuente: Elaboración propia sobre la base de la EPH, tercer trimestre de 2003.

Con un 3\% más que en 2011 (un 23\% para 2003 frente a un 20\% en 2011) la clase 1/4 presenta en 2003 un bajo volumen global de capital con una estructura patrimonial asociada a bajas calificaciones laborales, capital escolar de nivel primario e IPCF entre el $1^{\circ}$ y $2^{\circ}$ decil. La clase se asocia al cuentapropismo, la construcción y el servicio doméstico, con cierta precariedad laboral (no paga ni le descuentan obra social). Con una importante presencia de hogares con problemas de hacinamiento y recepción de ayuda material, este grupo presenta en su estructura patrimonial propiedades similares a las registradas en 2011. (Ver tabla 9).

Un comportamiento similar presentan los grupos altos dominantes, que pasan de un $14 \%$ en 2003 a un $17 \%$ en 2011. Este pequeño incremento no modifica las propiedades asociadas que definen la clase: altos ingresos, referentes con formación universitaria completa, cargos directivos, fundamentalmente en el Estado y condición de patrones o propietarios de empresas. Estas propiedades asociadas definen relacionalmente a las familias que conforma esta clase como un grupo con un alto volumen global de capital y una estructura patrimonial asociada a la propiedad de empresas o el control de la fuerza laboral (Patrones, Directivos y Jefes), calificaciones laborales profesionales y técnicas e Ingresos entre el $9^{\circ}$ y $10^{\circ}$ decil. 


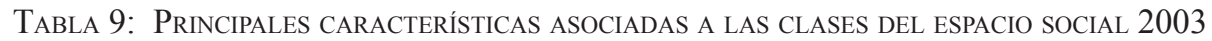

Clase media dominante (34\%)

Calificación Ocupacional: Técnica $(8,13)$

Nivel educativo: Univ. Incompleta $(7,01)$

Carácter Ocupacional: Educación $(6,47)$

Ingreso Per Cápita Familiar: $9^{\circ}$ Decil $(6,36)$

Nivel educativo: Univ. Completa $(5,36)$

Edad del RH: Hasta 34 años $(4,33)$

Ingreso Per Cápita Familiar: $5^{\circ}$ Decil $(4,08)$

Ámbito laboral: Estatal $(3,26)$

Nivel educativo: Sec. Completa $(2,56)$
Clase alta dominante (14\%)

Ingreso Per Cápita Familiar: $10^{\circ}$ Decil $(13,20)$

Calificación Ocupacional: Profesional $(11,01)$

Nivel educativo: Univ. Completa $(10,67)$

Jerarquía Ocupacional: Jefe $(5,61)$

Jerarquía Ocupacional: Dirección $(5,53)$

Categoría de Actividad: Patrón $(4,83)$

Ámbito laboral: Estatal $(3,29)$

Ingreso Ocupación Principal: $9^{\circ}$ Decil $(3,15)$

Calificación Ocupacional: Técnica $(2,58)$

Clase media dominada $(29 \%)$

Clase baja dominada $(23 \%)$

Ingreso Per Cápita Familiar: $3^{\circ}$ Decil $(11,82)$ Ingreso Per Cápita Familiar: $1^{\circ}$ Decil $(15,28)$

Nivel educativo: Sec. Incompleta $(7,87)$

Tipo de cobertura médica: No posee $(10,74)$

Calificación Ocupacional: Operativa $(5,74)$

Nivel educativo: Primaria Incompleta $(9,33)$

Nivel educativo: Primaria Completa $(5,24)$

Tipo de cobertura médica: No posee $(4,48)$

Recepción de mercaderías, ropa o alimentos $(7,58)$

Ingreso Total del RH: $7^{\circ}$ Decil $(4,20)$

Calificación Ocupacional: No Calificado $(5,19)$

Rama de Actividad: Servicio Doméstico $(4,66)$

Hogar con 6 personas o más $(3,98)$

Nivel educativo: Primaria Completa $(3,78)$

Rama de Actividad: Construcción (3,39)

Rama de Actividad: Construcción (2,92)

Jerarquía Ocupacional: Cuenta Propia $(2,98) \quad$ Más de 3 personas por ambiente exclusivo $(2,91)$

Fuente: Elaboración propia sobre la base de la EPH, tercer trimestre de 2003.

La lectura simultánea de las modalidades asociadas correspondientes a las clases medias de 2003, muestra que más allá de cierta modificación en el porcentaje de las familias que las componen (del $29 \%$ al $35 \%$ para la media dominada y del $34 \%$ al $29 \%$ para la 
media dominante), las estructuras patrimoniales de estos agrupamientos son similares en términos generales, lo que nos permite subrayar nuevamente la homología estructural entre los espacios de 2003 y 2011. Sin embargo, cabe señalar para el caso de la clase media dominada, que en el 2011 - Tabla 6- se incrementa la intensidad de la calificación laboral operativa del RH como propiedad de la clase, y su inserción en la rama de la industria (ausente en el 2003), lo que permitiría formular la hipótesis de que estas características pueden ser el resultado del proceso de industrialización de la última década.

Asimismo, éstas y otras diferencias en los valores test de las características que presentan cada una de las clases, pueden corresponderse a procesos sólo inteligibles a través de la complementación del análisis con otros métodos — especialmente cualitativos - que problematicen las estrategias de reproducción social y permitan la reconstrucción de trayectorias significativas (laborales y educativas, por ejemplo) en términos individuales, familiares, de clases y de fracciones de clase.

\section{Reflexiones finales: puntos de partida y apuestas}

Hemos presentado en este artículo una construcción del espacio social del Gran Córdoba (Argentina), tomando dos momentos similares correspondientes a los años 2003 y 2011, caracterizamos sus regiones y describimos las propiedades de las clases y fracciones que pueden identificarse. Esto constituyó un primer momento de una investigación acerca de las principales estrategias de reproducción social (laborales, educativas, habitacionales y de consumo cultural) que las distintas familias cordobesas ponen en marcha en la última década.

Está implícito aquí que antes de analizar las prácticas concretas, es necesario dar cuenta de la estructura que, en el marco de un conjunto de relaciones de dominación-dependencia (fundadas en la distribución desigual de los recursos económicos y culturales), de alguna manera dibujan los límites y las posibilidades que las estrategias tienen para desplegarse. Partir del volumen y estructura del capital (considerando a la familia como unidad de análisis) permite distinguir cuadros de disponibilidad de recursos diferenciados (Gutiérrez, 2004) y habilita a caracterizar detalladamente sistemas de estrategias diferentes, que rescatan la desigualdad y heterogeneidad de las diferentes posiciones sociales, buscando sus principales elementos explicativos en la estructura que conforman y en la trayectoria de la familia y del espacio.

La mirada teórica-metodológica que asumimos supone puntos de partida y representa un conjunto de apuestas de investigación que puntualizaremos como reflexiones finales.

Primer punto de partida: una manera de concebir la vida social y su reproducción. Como hemos mencionado más arriba, consideramos que ella es resultado de la acción dialéctica de estructuras y de agentes que, dotados de diferentes poderes y sin ser necesariamente conscientes de los mecanismos, la producen y la reproducen a través de 
las prácticas. A su vez, esas prácticas están ligadas a los distintos ámbitos de la vida social (trabajo, educación, vivienda, consumos culturales, etc.) y constituyen un conjunto sistemático de estrategias, a partir de las cuales las familias (cada familia, de las diferentes clases y fracciones de clase) enfrentan su existencia cotidiana y aseguran su reproducción social. Estas estrategias se definen (de manera no necesariamente consciente) a partir de los distintos tipos de recursos que se poseen (materiales y simbólicos, objetivos e incorporados): son esos recursos los que hacen posible la existencia de las estrategias y la especial modalidad de cada una de ellas; es necesario entonces conservarlos e, incluso, intentar incrementarlos. Por esta razón, en la medida en que tienden a reproducir el conjunto de los recursos disponibles, las estrategias de reproducción social tienen como orientación objetiva la conservación o la mejora de la posición en el espacio social: por ello, el mayor o menor "éxito" de las estrategias puestas en marcha por determinadas familias u otros grupos de agentes, depende también, indudablemente, del mayor o menor "éxito" que consigan los otros grupos sociales comprometidos en el mismo espacio social. Y como consecuencia de todos estos aspectos, consideramos que al producir y reproducir la vida social, agentes y estructuras producen y reproducen las condiciones que generan y sostienen las situaciones de desigualdad y las relaciones de dominación que las sustentan.

Segundo punto de partida: una manera de concebir la clase y con ello, de entrar en un debate que sigue siendo central en las ciencias sociales. Hemos expuesto brevemente nuestras herramientas conceptuales en el marco general y específico (los análisis en Latinoamérica y en Argentina) de los estudios actuales. Aquí hemos asumido que las clases sociales son, en primer lugar, "clases en el papel" — según la fórmula bourdieusiana-, construcciones operadas por el investigador.

Este trabajo representa también un conjunto de apuestas de investigación. Primero, una apuesta de articulación teórico-metodológica, que consiste en seleccionar aquellos métodos que nos permiten poner en marcha el pensamiento relacional que sustenta la mirada general y que nos habilita a hacer inteligibles las estructuras del mundo social que estamos estudiando. Hemos mostrado aquí la utilidad de la combinación del análisis de las correspondencias con el de la clasificación jerárquica ascendente, a través de un software específico, señalando al mismo tiempo que esta tarea conlleva un conjunto de decisiones teóricas tendientes a coordinar estos métodos con los objetivos, hipótesis y unidades de análisis de nuestro estudio, a la vez que con las posibilidades que brindan las fuentes de información disponibles.

En ese sentido, hacemos también una apuesta por el uso de la información producida por nuestro propio sistema estadístico nacional, en este caso, como hemos mostrado, de la EPH para el Gran Córdoba. Se trata de una decisión práctica, sin duda, ante la imposibilidad de poner en marcha encuestas ad hoc que impliquen una muestra representativa, a gran escala, y con la periodización que requiere el estudio. Se trata también de una decisión ética y política asociada a las discusiones que insume su uso, respecto 
a las unidades de análisis que están presentes en esta fuente y las que son pertinentes en nuestro estudio, a las variables que captura la EPH y aquellas que remiten a los diferentes capitales que estructuran el espacio social general, a la elección de un "referente de hogar" que puede o no coincidir con el "jefe de hogar" que registra la encuesta, etc. Estas son algunas de las cuestiones que nos permiten indicar límites y posibilidades de las capturas periódicas de nuestro sistema estadístico y con ello, intentar incidir en su diseño e implementación.

Forma parte también de la apuesta política el desarrollo de este tipo de estudios en el interior del país, y lo es en el sentido de cubrir la relativa carencia de investigaciones sobre la estructura social y la dinámica de las clases fuera del área metropolitana de Buenos Aires, que ha sido señalada como "una de las principales deudas de las ciencias sociales con el conocimiento de la realidad argentina" (Del Cueto y Luzzi, 2008: 8).

Finalmente, destaquemos que esta propuesta constituye también una apuesta en la articulación de las dimensiones cuantitativa y cualitativa de la investigación. Esta manera de construir el espacio social cordobés es un insumo necesario para dar cuenta de las estrategias de reproducción social de las familias en el marco de la estructura de relaciones en las que se hallan inmersas, pero no podemos olvidar que esas relaciones de fuerza implican además relaciones de sentido, susceptibles de ser captadas a través de otros métodos de recolección y análisis. Lo presentado aquí nos permite identificar y seleccionar los casos típicos que, a modo de parangones, posibilitan explicar y comprender las prácticas y representaciones concretas y reconstruir trayectorias que, siendo individuales y/o familiares, son a la vez representativas de colectivos como las clases o fracciones de clase.

\section{Bibliografía}

Baranger, D. (1993): Construcción y análisis de datos, Posadas, Editorial Universitaria de Misiones.

BENZÉCRI, J. P. et al. (1973): L'analyse des donnés. Tome 1: La taxonomie, Tome 2: L'analyse des correspondances, Paris, Dunod.

Bourdieu, P. (1988): La Distinción: Criterios y bases sociales del gusto, Madrid, Taurus.

Bourdieu, P. (1990): “Espacio social y génesis de las clases”, en P. Bourdieu, Sociología y Cultura, México, Grijalbo.

Bourdieu, P. (1991): El sentido práctico, Madrid, Taurus.

Bourdieu, P. (1997): "Espacio social y espacio simbólico. Introducción a una lectura japonesa de La distinción", en P. Bourdieu, Capital cultural, escuela y espacio social, México, Siglo XXI.

Bourdieu, P. (1999): "Efectos de lugar", en P. Bourdieu et al., La miseria del mundo, Buenos Aires, FCE. 
Bourdieu, P. (2013): La nobleza de Estado. Educación de élite y espiritu de cuerpo, Buenos Aires, Siglo XXI.

Bourdieu, P. et al. (1975): El oficio de sociólogo, Buenos Aires, Siglo XXI.

Capdevielle, J. (2012): "Redes religiosas y superación de la pobreza: potencialidades, límites y contradicciones”, Humanitas: Revista de Investigación, 9 (9), pp. 131- 144.

Crompton, R. (1997): Clase y estratificación: Una introducción a los debates actuales, Madrid, Tecnos.

Del Cueto, C.M. y Luzzi, M. (2008): Rompecabezas. Transformaciones en la estructura social argentina (1983 - 2008), Buenos Aires, Universidad Nacional General Sarmiento / Biblioteca Nacional.

Devine, F. (2005): "Middle-Classs Identities in the United States", en Devine, Savage, Scott \& Crompton (eds.), Rethinking Class. Culture, Identities \& Lifestyle. Bakingstone, Palgrave Macmillan.

Devine, F. \& M. Savage (2005): "The Cultural Turn, Sociology and Class Analysis", en Devine, Savage, Scott \& Crompton (eds.), Rethinking Class. Culture, Identities \& Lifestyle. Bakingstone, Palgrave Macmillan.

EspinozA, V. (2006): "La movilidad ocupacional en el Cono Sur: Oportunidades y desigualdad social”, Revista de Sociología, 20, pp. 131-146.

FACHelli, S. (2012): "Desigualdad y estratificación social en la Argentina”, en S. Fachelli et. al., Desigualdad y diversidad en América Latina: hacia un análisis tipológico comparado, Buenos Aires / Madrid, IIPE / UNESCO / OEI.

Freyre, M.L. (2013): "Políticas de empleo: Programas sociales con condicionalidad: el caso del Plan Jefes y Jefas de Hogar Desocupado en una localidad pobre de Córdoba", Revista Trabajo y Sociedad, 21, pp. 495-514.

Germani, G. (1955): Estructura social de la Argentina, Buenos Aires, Raigal.

GidDEns, A. (1983) La estructura de clases en las sociedades avanzadas, Madrid, Alianza.

GidDENs, A. (1987): Las nuevas reglas del método sociológico, Buenos Aires, Amorrortu.

GidDEns, A. (1995): La constitución de la sociedad, Buenos Aires, Amorrortu.

Goldthorpe, J. (1994): "Sobre la clase de servicio, su formación y su futuro", en J. Carabaña y A. De Francisco (Comps.), Teorías contemporáneas de las clases sociales, Madrid, Editorial Pablo Iglesias.

Gutiérrez, A. (2004): Pobre' como siempre... Estrategias de reproducción social en la pobreza, Córdoba, Ferreyra Editor. 
GutiérRez, A. (2005): Estratégias, capitais e redes: elementos para a análise da pobreza urbana, en Cattani, A. y L. Mota Díaz, (eds.), Desigualdades na América Latina. Novas perspectivas analiticas (pp. 13-49). Rio-Grande do Sul: Universidade Federal do Rio Grande do Sul / Centro de Estudios sobre Marginación y Pobreza del Estado de México / Asociación Latinoamericana de Sociología.

Gutiérrez, A. (2007): "Herramientas teórico-metodológicas de un análisis relacional para los estudios de la pobreza", Ciencia, Docencia y Tecnología, 35, pp. 15-33.

GutiérRez, A. (2011): “Clase, espacio social y estrategias. Una introducción al análisis de la reproducción social en Bourdieu", en P. Bourdieu, Las estrategias de la reproducción social, Buenos Aires, Siglo XXI.

Gutiérrez, A. y H. Mansilla (2013): "El espacio social y su reproducción: aspectos teórico metodológicos y fuentes secundarias", XXIX Congreso de la Asociación Latinoamericana de Sociología - ALAS- 2013 "Crisis y Emergencias Sociales en América Latina", Santiago de Chile.

JiMÉNEZ Zunino, C. (2011a): “¿De dónde vienen? Las estrategias migratorias de reproducción social”, Estudios Migratorios Latinoamericanos, 71, pp. 433-462.

JimÉNEZ Zunino, C. (2011b). ¿"Empobrecimiento o desclasamiento? La dimensión simbólica de la desigualdad social", Trabajo y sociedad, 17 (15), pp. 49-65.

Jorrat, J. R. (2005): “Aspectos descriptivos de la movilidad intergeneracional de clase en Argentina: 2003-2004", Laboratorio/n Line, 17/18,

$<$ http://www.catedras.fsoc.uba.ar/salvia/lavbo/textos/17-18_1.htm> Consultado: 22/08/2013.

Kessler, G. y V. Espinoza (2003). Movilidad social y trayectorias en Buenos Aires: Rupturas y algunas paradojas, Santiago de Chile, CEPAL.

LÉBART, L. (1989): "Stratégies du traitement des données d'enquête", Revue du Modulad, 3, pp. 21-29.

Mansilla, H. (2011): Nuevos Consumos Culturales. Tecnologías y bienes simbólicos. Aportes teórico-metodológicos, Villa María, EDUVIM.

Moscoloni, N. (2005): La nube de datos, Rosario, UNR Editora.

Torrado, S. (1998): Familia y diferenciación social: Cuestiones de método, Buenos Aires, Eudeba.

Vester, M. (2005): “Class and Culture un Germany”, en Devine, Savage, Scott \& Crompton (eds.), Rethinking Class. Culture, Identities \& Lifestyle, Bakingstone, Palgrave Macmillan.

Wacquant, L. (1991): "Making Class: The Middle Class(es) in Social Theory and Social Structure", en R. Levine, S. Mcnall \& R. Fantasia (eds.), Bringing Class Back In. Contemporary and Historical Perspectives, USA, Westview Press. 
Weininger, E.B. (2005): "Foundations of Pierre Bourdieu's class analysis", en E.O. Wright (ed.) Approaches to Class Analysis, Cambridge, University Press.

Wright, E.O. (1994): "Reflexionando, una vez más, sobre el concepto de estructura de clases", en J. Carabaña y A. De Francisco (comps.), Teorías contemporáneas de las clases sociales, Madrid, Editorial Pablo Iglesias. 